\title{
Symptomatic congenital cytomegalovirus disease following non-primary maternal infection: a retrospective cohort study
}

\author{
Eran Hadar ${ }^{1,2^{*}}$, Elizabeta Dorfman², Ron Bardin ${ }^{1,2}$, Rinat Gabbay-Benziv ${ }^{1,2}$, Jacob Amir ${ }^{2,3}$ and Joseph Pardo ${ }^{1,2}$
}

\begin{abstract}
Background: Scarce data exist about screening, diagnosis and prognosis of non-primary Cytomegalovirus (CMV) during pregnancy. We aimed to examine antenatal diagnosis of maternal non-primary CMV infection and to identify risk factors for congenial CMV disease.

Methods: Retrospective cohort of 107 neonates with congenital symptomatic CMV infection, following either primary $(n=95)$ or non-primary $(n=12)$ maternal CMV infection. We compared the groups for the manifestations and severity of congenial CMV disease, as well as for possible factors associated with the risk of developing CMV related infant morbidity.

Results: Disease severity is not similar in affected newborns, with a higher incidence of abnormal brain sonographic findings, following primary versus non-primary maternal CMV infection (76.8\% vs. 8.3\%, $p<.001$ ). Symptomatic congenital CMV disease following a non-primary infection is more frequent if gestational hypertensive disorders and/or gestational diabetes mellitus have ensued during pregnancy (33.3\% vs. 9.9\%, $p<0.038$ ), as well as if any medications were taken throughout gestation ( $50 \%$ vs. $16.8 \%, p<0.016)$. CMV-IgM demonstrates a low detection rate for non-primary maternal infection during pregnancy compared to primary infection ( $25 \%$ vs. $75.8 \%, p=0.0008$ ).
\end{abstract}

Conclusion: Non-primary maternal CMV infection has an impact on the neonate. Although not readily diagnosed during pregnancy, knowledge of risk factors may aid in raising clinical suspicion.

Keywords: Cytomegalovirus, Non-primary, Secondary, Diagnosis, Congenital, Risk factors

\section{Background}

Cytomegalovirus (CMV) is a common congenital infection, diagnosed among $0.3-2 \%$ of newborns [1]. The prevalence and severity of congenital CMV infection varies according to the timing of infection, the presence of preexisting immunity and other contributing factors [2]. Secondary, or non-primary, maternal CMV infection occurs when prior maternal immunity exists. It can emerge following latent CMV reactivation or by a new viral strain reinfection [3]. Following a non-primary maternal infection the vertical transmission rate of CMV is reported to be $0.2-1 \%$ [4] and congenital symptomatic disease is diagnosed in less than $1 \%$, among fetuses infected in-utero [5]. Moreover,

\footnotetext{
* Correspondence: eranh42@gmail.com

${ }^{1}$ Helen Schneider Hospital for Women, Rabin Medical Center, 39 Zabotinski St., Petach-Tikva 49100, Israel

${ }^{2}$ Sackler Faculty of Medicine, Tel Aviv University, Tel Aviv, Israel

Full list of author information is available at the end of the article
}

congenital CMV has a clinical spectrum ranging from asymptomatic infection to a severe disease, including multiple possible manifestations of petechiae, jaundice, hepatitis, chorioretinitis, microcephaly, growth restriction, hepatosplenomegaly; as well as short and long term squelae of hearing loss and neurodevelopmental deficits [6]. It is debatable whether prior immunity infers complete or partial protection against CMV related morbidity [7, 8], while others have reported that disease manifestations, severity and long term prognosis are similar between primary and non-primary infections [9-11].

The rate of congenital infection following non-primary maternal CMV infection is extremely low compared to the incidence subsequent to primary infection [12, 13]. Nevertheless, non-primary maternal CMV infection has been previously reported as a cause of congenital symptomatic disease $[14,15]$. In the United States, only $25 \%$ 
of infants with congenital CMV infection were attributable to primary maternal infection, while $75 \%$ were born to mothers with non-primary infections, following either reactivation or reinfection with different viral strains [16]. Moreover, in a recent population-based prediction model, it was estimated that non-primary infections account for the majority of CMV-related hearing losses [17].

Paucity of reports exist on appropriate tools to screen and diagnose non-primary gestational CMV and to detect women who are at risk for delivering CMV symptomatic infants, following a non-primary infection. Therefore, we aimed to examine the antenatal diagnosis of maternal non-primary CMV infection and to identify antenatal risk factors for congenial symptomatic CMV among neonates.

\section{Methods}

We conducted a retrospective cohort study of all neonates that were referred for follow up due to congenital CMV infection. The study was approved by the Rabin medical center institutional review board.

\section{Study population}

The study population included all neonates born from January 2006 to December 2013, who were referred for follow up due to congenital symptomatic CMV infection. The study was conducted at the Rabin medical center, which is a university-affiliated, tertiary medical center which serves as a referral center for both high risk pregnancies (Helen Schneider Hospital for Women) and pediatric patients (Schneider Children's Medical Center of Israel).

Eligibility criteria for the study included all the newborns with a positive urinary culture or PCR for CMV, taken shortly after birth and up to two weeks following delivery. Those with insufficient data as to antenatal and/or neonatal care and serological data were excluded from the study, as well as those newborns with a congenital asymptomatic infection. The included population was sub-divided to symptomatic neonates following either non-primary maternal CMV infection (study group) versus primary infection (control group).

Symptomatic newborns included those with any one of the following symptoms or signs - small for gestational age (SGA, defined as birthweight below the 3rd percentile for gestational age at delivery), chorioretinitis, abnormal brain sonography (defined as the presence of any of the following: calcification, periventricular hyperecogenicity, ventriculomegaly, subependymal cysts and lenticular-striated vasculopathy (LSV)), microcephaly (defined as age appropriate head circumference below two standard deviations), pathological audio screening, sensorineural hearing loss (SNHL) detected by abnormal brainstem-evoked response audiometry test (BERA) and that the treating physician has decided to start antiviral treatment for any one of the above mentioned signs and symptoms.

\section{Maternal and neonatal follow up}

The diagnosis of primary maternal CMV infection was established according to the following criteria: women who seroconvert from negative to positive CMV-IgG during pregnancy; or if prior serology for CMV was not available - low CMV-IgG avidity and positive specific CMV-IgM were also considered diagnostic for primary maternal CMV infection. Non-primary CMV infection was considered when pre-gestational maternal immunity was established from previous laboratory results, i.e. positive CMV-IgG documented prior to pregnancy.

Mothers who were diagnosed during pregnancy with gestational CMV infection were offered prenatal diagnosis with amniocentesis. The procedure was performed beyond 21 completed weeks of gestation and at least 7 weeks after the presumed timing of maternal CMV infection. Following amniocentesis, if fetal infection was documented and pregnancy was not terminated, serial ultrasound (US) assessments were recommended every 3-4 weeks and magnetic resonance imaging (MRI) was suggested at 32-34 weeks. Fetal imaging results - by either MRI or US - were considered as abnormal, an related to CMV, if they included any one of the following findings: fetal growth restriction (defined as estimated fetal weight below the 3rdpercentile for gestational age), ventriculomegaly, microcephaly, central nervous system (CNS) calcification, periventricular hyperecogenicity, subependymal cysts, increased white matter signal and abnormal gyration or hyperechogenic bowel.

Congenital CMV infection in the newborn was established by a CMV positive urinary shell vial culture or PCR, preformed within $48 \mathrm{~h}$ after birth and up to 2 weeks following delivery. Neonates with a positive culture underwent a thorough evaluation, upon admission, comprised of: BERA, ocular funduscopy, brain ultrasound and blood work-up for complete blood count, liver enzymes and bilirubin. CNS sonographic signs related to neonatal CMV included: calcifications, periventricular hyperecogenicity, ventriculomegaly, pseudocysts and LSV. Neonates diagnosed with CMV infection after birth were followed at our clinic once a month until 3 months of age and every 3-6monthsthereafter. A full physical examination, including neurological and developmental assessments, was performed at each visit. Blood count was tested every other week up to 3 months of age and then during subsequent clinical visits. BERA was performed in the first month, and thereafter at least twice in the first year of life, with follow up periodic examinations every 6 months to age 2 years. Behavioral learning evaluation was preformed every 6 months up to age 4 . 


\section{Data extraction and definitions}

We extracted maternal, fetal and neonatal data from medical charts, delivery records and computerized databases of laboratory and imaging data. The extracted data for the study included: maternal demographics, medical and obstetrical history, maternal occupation (high risk occupations for CMV exposure were defined as kindergarten teachers, day care workers and medical professionals), pregnancy complications (Hypertensive disorders, gestational diabetes, preterm delivery prior to 37 weeks of gestation and maternal febrile illness during pregnancy), use of medication throughout pregnancy and delivery outcome (mode and time of delivery, fetal gender, Apgar score and birthweight). Laboratory and imaging data was also extracted, including: complete maternal serologic data (IgG, IgM and IgG avidity), liver enzyme testing, amniocentesis and imaging (US and/or MRI) results for CMV obtained during pregnancy and neonatal follow up.

\section{Statistical analysis}

Statistical analysis was performed using the Statistical Package for Social Science (SPSS, IBM software version 21.0, Chicago, IL, USA). Categorical variables were compared using Fischer's exact or Pearson Chi-square tests. Continuous variables were analyzed by One Way Analysis of Variance (ANOVA). A probability value below 0.05 was considered significant.

\section{Results}

A total of 173 newborns with proven congenital CMV infection were evaluated, of which six were excluded due to lack of antenatal data and 60 due to a neonatal asymptomatic infection. Therefore, 107 newborns with symptomatic congenital CMV disease were available for final analysis - $12(11.2 \%)$ newborns were symptomatic following non-primary CMV maternal infection (study group) and $95(88.8 \%)$ were affected following primary CMV maternal infection (control group).

The manifestations of the congenital CMV disease among the neonates, in each of the groups, are presented in Table 1. Our results demonstrate that symptomatic newborns following maternal non-primary CMV infection were significantly less likely to present an abnormal brain sonography compared to neonates with congenital CMV disease following primary CMV infection $(8.3 \%$ vs. $76.8 \%, p<.001)$.

\section{Maternal factors (Table 2)}

Women giving birth to a symptomatic newborn, following a non-primary CMV infection during pregnancy, were more likely to have medical complications during pregnancy $(33.3 \%$ vs. $9.9 \%, p<0.038)$ and were more likely to have used medications during pregnancy (50\% vs. $16.8 \%, p<0.016)$ compared to women delivering
Table 1 CMV related findings among symptomatic neonates, following primary versus non-primary maternal infection

\begin{tabular}{lcll}
\hline & $\begin{array}{l}\text { Primary }(n= \\
95)\end{array}$ & $\begin{array}{l}\text { Non-primary }(n= \\
12)\end{array}$ & $\begin{array}{l}P \\
\text { value }\end{array}$ \\
\hline Small for gestational age & $17(17.9 \%)$ & $1(16.6 \%)$ & 0.686 \\
Chorioretinitis & $5(5.3 \%)$ & 0 & 0.999 \\
Abnormal brain sonography & $73(76.8 \%)$ & $1(8.3 \%)$ & $<.0001$ \\
Microcephaly & $5(5.3 \%)$ & $1(8.3 \%)$ & 0.904 \\
Pathological audio screening & $18(18.9 \%)$ & $3(25 \%)$ & 0.7 \\
test & & & 0.117 \\
Abnormal BERA & & & 0.498 \\
\multicolumn{1}{c}{ At admission } & $32(33.7 \%)$ & $7(58.3 \%)$ & \\
\multicolumn{1}{l}{ At follow up } & $40(42.1 \%)$ & $7(58.3 \%)$ & \\
\hline
\end{tabular}

Data presented as $\mathrm{n}(\%)$

CMV Cytomegalovirus, BERA Brainstem Evoked Response Audiometry

after a primary CMV infection. Serological diagnosis of maternal infection was established during pregnancy in only 3 women among the non-primary infection subgroup. We found that the likelihood of positive IgM-CMV was significantly lower ( $25 \%$ vs. $75.8 \%, p=0.0008)$ among the non-primary subgroup compared to the primary subgroup, respectively.

Table 2 Maternal characteristics among symptomatic neonates, following primary versus non-primary maternal infection

\begin{tabular}{llll}
\hline & $\begin{array}{l}\text { Primary } \\
(n=95)\end{array}$ & $\begin{array}{l}\text { Non-primary } \\
(n=12)\end{array}$ & $P$ value \\
\hline Maternal age, years & $29.12 \pm 5.12$ & $27.77 \pm 4.60$ & 0.452 \\
Maternal chronic disease & $14(15.1 \%)$ & $3(27.3 \%)$ & 0.382 \\
High professional risk & $19(20 \%)$ & $2(16.7 \%)$ & 0.999 \\
Obstetrical history & & & \\
Parity & $2.24 \pm 1.21$ & $2.16 \pm 1.69$ & 0.848 \\
Live births & $2.03 \pm 0.93$ & $2.0 \pm 1.41$ & 0.918 \\
Abortions & $0.21 \pm 0.74$ & $0.17 \pm 0.39$ & 0.847 \\
Parity, with living children $(\geq 1)$ & $75(78.9 \%)$ & $7(58.3 \%)$ & 0.146 \\
Complications during pregnancy & $9(9.9 \%)$ & $4(33.3 \%)$ & 0.038 \\
Medication use during pregnancy & $16(16.8 \%)$ & $6(50 \%)$ & 0.016 \\
Febrile disease at CMV diagnosis & $31(38.8 \%)$ & $1(8.3 \%)$ & 0.103 \\
Gestational age at CMV diagnosis: & $19.50 \pm 8.38$ & $19.16 \pm 13.51$ & 0.928 \\
$1^{\text {st }}$ trimester & $44(46.3 \%)$ & $3(25 \%)$ & 0.318 \\
$2^{\text {nd }}$ trimester & $42(44.2 \%)$ & $1(8.3 \%)$ & 0.03 \\
$3^{\text {rd }}$ trimester & $9(9.5 \%)$ & $2(16.7 \%)$ & 0.356 \\
Maternal serology at CMV diagnosis & & & \\
Positive lgG & $81(85.3 \%)$ & $12(100 \%)$ & $<.0001$ \\
IgG Seroconversion & $79(83.1 \%)$ & - & - \\
Positive lgM & $72(75.8 \%)$ & $3(25 \%)$ & 0.0008 \\
Low avidity lgG & $33(34.7 \%)$ & - & - \\
\hline Data prent & & & \\
\hline
\end{tabular}

Data presented as $\mathrm{n}(\%)$ or mean \pm 1 Standard Deviation CMV Cytomegalovirus 


\section{Fetal factors (Table 3 )}

Mothers exposed to non-primary CMV infection were less likely to perform amniocentesis than those with primary infection $(16.7 \%$ vs. $49.5 \%, p=0.035)$. Also, the chance of positive CMV in amniotic fluid analysis among fetuses exposed to non-primary CMV infections was lower than those in the primary subgroup $(0 \%$ vs. $89.4 \% \cdot p=0.018$ ).

\section{Discussion}

Our study was a retrospective cohort analysis of 107 newborns, stratified according to preexisting maternal immunity. Our main results indicate that disease manifestation and severity are not similar between groups; antenatal risk factors for a congenital CMV disease following a non-primary infection include the occurrence of pregnancy complications and the use of medication during pregnancy; and that CMV-IgM demonstrates low detection rate for non-primary maternal infection.

Previous studies have reported contradicting findings, whether CMV following non-primary infection is associated with a higher or lower incidence $[18,19]$ and severity [7-11] of congenital CMV. Boppana et al. [9], in a similarly designed study to our own, compared 47 symptomatic neonates - 12 following non-primary CMV and 35 following primary CMV infection. They demonstrated similar rates of CMV related symptoms (SGA, jaundice, hepatosplenomegaly, microcephaly, chorioretinitis and abnormal hearing screen) and comparable CMV disease severity among the groups. Townsend et al. [10] and Ross et al. [11] have

Table 3 Fetal characteristics among symptomatic neonates, following primary versus non-primary maternal infection

\begin{tabular}{llll}
\hline & $\begin{array}{l}\text { Primary } \\
(n=95)\end{array}$ & $\begin{array}{l}\text { Non-primary } \\
(n=12)\end{array}$ & $P$ value \\
\hline Fetal gender & $51(53.7 \%)$ & $5(41.7 \%)$ & 0.544 \\
$\quad$ Male $(n=56)$ & $44(46.3 \%)$ & $7(58.3 \%)$ & 0.544 \\
$\quad$ Female $(n=51)$ & & & \\
Mode of delivery & $64(67.4 \%)$ & $8(66.7 \%)$ & 0.999 \\
$\quad$ Vaginal delivery & $6(6.3 \%)$ & $2(16.7 \%)$ & 0.22 \\
$\quad$ Instrumental delivery & $25(26.3 \%)$ & $2(16.7 \%)$ & 0.726 \\
$\quad$ Cesarean delivery & $38.92 \pm 1.56$ & $39.00 \pm 2.00$ & 0.882 \\
Gestational age at delivery, weeks & $3070 \pm 530$ & $3167 \pm 817$ & 0.607 \\
Birth weight, grams & $33.71 \pm 1.63$ & $33.77 \pm 1.93$ & 0.909 \\
Neonatal head circumference, cm & $47(49.5 \%)$ & $2(16.7 \%)$ & 0.035 \\
Amniocentesis preformed during & $42(89.4 \%)$ & 0 & 0.018 \\
pregnancy: & $11(11.6 \%)$ & $4(33.4 \%)$ & 0.114 \\
$\quad$ Positive for CMV & $11(11.6 \%)$ & 0 & 0.608 \\
\hline Abnormal findings on fetal US & &
\end{tabular}

Data presented as $\mathrm{n}(\%)$ or mean \pm 1 Standard Deviation

CMV Cytomegalovirus, US Ultrasound, MRI Magnetic Resonance Imaging shown, respectively, that hearing loss and overall long term sequelae have similar incidence in primary and nonprimary CMV symptomatic newborns. Contradictory to these reports, Fowler et al. [8], comparing 125 neonates born following primary maternal infection vs. 64 neonates born following non-primary infection demonstrated that only those with primary infection were symptomatic at birth - including jaundice, petechiae, hepatosplenomegaly, SGA, microcephaly, hydranencephaky and death. Also, that late onset handicaps and squelae were apparent among $25 \%$ of neonates born following primary infection, versus only $8 \%$ in neonates of the non-primary CMV group. Our study partly supports all these findings, as we demonstrated similar rates of SGA, microcephaly, chorioretinitis, pathological audio screening test and BERA. However, we found higher rates of abnormal brain sonography in the primary CMV group, a sign which has not been evaluated specifically in previous studies.

The majority of current literature on the prediction of symptomatic CMV disease among infected fetuses is focused on the timing of maternal infection during gestation and the presence of abnormal findings in fetal imaging, as predictors of symptomatic congenital disease. Our study is the first to demonstrate that nonsonographic antenatal factors may be associated with an increased risk to deliver an affected newborn. Our results support the possibility that the occurrence of pregnancy complications - specifically hypertensive disorders and gestational diabetes - as well as the use of chronic medication during pregnancy is associated with an increased risk of delivering a CMV symptomatic newborn, following a non-primary CMV maternal infection. There is paucity of data on antenatal risk factors for symptomatic disease, and no data focusing on the subgroup of non-primary CMV. Boppana et al. [9], demonstrated no difference among primary versus non-primary groups, with regards to parity and the number of children of the infected mothers. Others [20] have reported that young age (below 20 years), non-white race and low-income status are associated with an increased risk for delivering a neonate exhibiting CMV related morbidity - however, no distinction was made with regard to maternal immunity status during pregnancy in this study. In a previously published study [21], we demonstrated that antenatal risk factors for CMV congenital disease following primary maternal infection may include - young maternal age and a high risk occupational exposure. Accordingly, we speculate that primary infection is related to external factors, conferring a higher exposure risk of the mother to new onset CMV infection, while a nonprimary infection is associated with internal factors, exposing the mother to either reactivation or reinfection with CMV. Although the mechanism for such an association is unknown, we speculate that it is may be 
associated with some degree of interference to the immune system, leading to higher maternal susceptibility to latent viral reactivation. Although, for either primary or non-primary infection, the final common pathway is a higher viral load during gestation, allowing for greater viral inoculums to reach the placenta and fetus, consequently increasing the risk for symptoms at birth.

Serological diagnosis of non-primary CMV infection in the mother is problematic. Due to the asymptomatic nature of maternal CMV infection in the majority of cases, clinical suspicion of CMV infection rarely rises. Maternal CMV infection is usually diagnosed by routine screening in sero-negative women or may be suspected due to abnormal sonographic findings. In our cohort maternal serology was examined for the presence of CMV-IgM in only 6 cases. The trigger for serological testing was due to either febrile illness $(n=1)$, suspicious sonographic finding $(n=4)$ or routine testing $(n=1)$. Among those 6 mothers, CMV-IgM was positive in only half (50\%). This rate was significantly lower than the rate of positive CMV-IgM (75.8\%) among mothers in the primary infection. The low rate of positive CMV-IgM among mothers with non-primary CMV infection is supported by other small studies [22, 23]. Kyriazopoulou et al. [22] studied 32 women immunized to CMV with normal pregnancy course and no suspicious ultrasound findings. Although, none of the women had any serological evidence of maternal non-primary CMV infection, they detected $4(12.5 \%)$ positive CMV results in amniotic fluid or fetal blood sampling. In another study, Zalel at al. [23] studied 6 pregnant women who were referred due to abnormal sonographic findings, suggestive of in-utero CMV related morbidity. All women were exposed to CMV prior to pregnancy and none had serological evidence of non-primary infection at workup. However, all women had positive CMV in amniotic fluid analysis. These studies, in concordance with our current study, demonstrate a low detection rate for non-primary CMV infection by CMV-IgM testing.

A major strength of our study is that in contrast to other studies, the immune status of all participating women was known and ascertained by documented serological examinations. Indeed, the study is limited due to its retrospective nature, with a limited number of participants, especially in the non-primary CMV group, where only symptomatic newborns were referred for follow-up. Such a referral is routinely recommended when suspected finding arise during pregnancy or at the immediate postpartum period due to abnormal sonographic findings, clinical findings and abnormal audio screening tests - this may explain the high rate of hearing loss in the study. Importantly, the primary CMV infection group includes only newborns to mothers who elected to continue their pregnancy despite proven inutero infection or alarming findings on prenatal imaging.
Due to lack of data we could not determine whether the non-primary infection was a result of reactivation or reinfection, both possible causes of congenital CMV [24], although no data exist whether one causes more serious morbidity than the other [7].

\section{Conclusion}

The true incidence and impact of recurrent maternal CMV infection remains elusive, with questions as to risk factors, screening and diagnosis. However, it seems that the incidence of symptomatic non-primary infection is low and somewhat limited to pregnancies complicated by maternal morbidity. When such fetal-neonatal infection occurs, it might be with less severe sequelae compared to neonates following primary maternal infection. Our results bring to focus the importance of sonographic follow up during pregnancy, which may provide clues for the possibility of congenital symptomatic CMV fetal infection. When such relevant sonographic markers are detected, the absence of presumed serological evidence (i.e. positive IGM), should not prevent further diagnostic measures such as amniocentesis and further fetal imaging such as a detailed targeted sonography and MRI. This also raises the issue of newborn routine screening as a major tool for prompt and timely diagnosis of congenital CMV. Such early detection may allow workup, treatment and future explorations to prevent long term handicap. Undoubtedly, further large scale studies are needed, focusing on prevention of non-primary CMV among gravidas, fetal diagnosis during pregnancy and screening for congenital CMV among neonates, as well as possible interventions to prevent long term poor prognosis among congenitally infected neonates.

\section{Abbreviations}

BERA: Brainstem-evoked response audiometry; CMV: Cytomegalovirus; CNS: Central nervous system; LSV: Lenticular striate vasculopathy; MRI: Magnetic resonance imaging; SGA: Small for gestational age; SNHL: Sensorineural hearing loss; US: Ultrasound

\section{Acknowledgments}

Not applicable

Funding

Not applicable, no funding was received to support this study.

\section{Availability of data and materials}

The datasets during and/or analyzed during the current study are available from the corresponding author on reasonable request.

\section{Authors' contributions}

EH made substantial contributions to conception and design, acquisition of data and analysis and interpretation of data, as well as involved in drafting the manuscript. ED made substantial contributions to conception and design, acquisition of data and analysis and interpretation of data, as well as involved in drafting the manuscript. RGB made substantial contributions to conception and design, analysis and interpretation of data, as well as involved in drafting the manuscript. RB made substantial contributions to conception and design, analysis and interpretation of data, as well as involved in drafting the manuscript. JA

made substantial contributions to conception and design, acquisition of data and analysis and interpretation of data, as well as involved in drafting the manuscript. JP made substantial contributions to conception and design, acquisition of data 
and analysis and interpretation of data, as well as involved in drafting the manuscript. All authors gave final approval of the version to be published, and agreed to be accountable for all aspects of the work.

\section{Competing interests}

The authors declare that they have no competing interests.

\section{Consent for publications}

Not applicable.

\section{Ethics approval and consent to participate}

The study was approved by the Rabin medical center institutional review board. Obtaining informed consent for participation was waived by the review board.

\section{Author details}

${ }^{1}$ Helen Schneider Hospital for Women, Rabin Medical Center, 39 Zabotinski St., Petach-Tikva 49100, Israel. ${ }^{2}$ Sackler Faculty of Medicine, Tel Aviv University, Tel Aviv, Israel. ${ }^{3}$ Pediatrics C Department, Schneider Children's Medical Center of Israel, Petach-Tikva, Israel.

Received: 3 July 2016 Accepted: 22 December 2016

Published online: 05 January 2017

\section{References}

1. Stagno S, Whitley RJ. Herpesvirus infections of pregnancy. Part I: cytomegalovirus and Epstein-Barr virus infections. N Engl J Med. 1985;313(20):1270-4.

2. Stagno S, Pass RF, Dworsky ME, Alford CA. Maternal cytomegalovirus infection and perinatal transmission. Clin Obstet Gynecol. 1982;25(3):563-76.

3. Boppana SB, Rivera LB, Fowler KB, Mach M, Britt WJ. Intrauterine transmission of cytomegalovirus to infants of women with preconceptional immunity. N Engl J Med. 2001;344(18):1366-71.

4. Raynor BD. Cytomegalovirus infection in pregnancy. Semin Perinatol. 1993;17(6):394-402.

5. Stagno S, Pass RF, Cloud G, Britt WJ, Henderson RE, Walton PD, et al. Primary cytomegalovirus infection in pregnancy. Incidence, transmission to fetus, and clinical outcome. JAMA. 1986;256(14):1904-8.

6. Boppana SB, Ross SA, Fowler KB. Congenital cytomegalovirus infection: clinical outcome. Clin Infect Dis. 2013;57 Suppl 4:S178-81.

7. Stagno S, Pass RF, Dworsky ME, Henderson RE, Moore EG, Walton PD, et al. Congenital cytomegalovirus infection: the relative importance of primary and recurrent maternal infection. N Engl J Med. 1982;306(16):945-9.

8. Fowler KB, Stagno S, Pass RF, Britt WJ, Boll TJ, Alford CA. The outcome of congenital cytomegalovirus infection in relation to maternal antibody status. N Engl J Med. 1992;326(10):663-7.

9. Boppana SB, Fowler KB, Britt WJ, Stagno S, Pass RF. Symptomatic congenital cytomegalovirus infection in infants born to mothers with preexisting immunity to cytomegalovirus. Pediatrics. 1999;104(1 Pt 1):55-60.

10. Townsend CL, Forsgren M, Ahlfors K, Ivarsson SA, Tookey PA, Peckham CS. Long-term outcomes of congenital cytomegalovirus infection in Sweden and the United Kingdom. Clin Infect Dis. 2013;56(9):1232-9.

11. Ross SA, Fowler KB, Ashrith G, Stagno S, Britt WJ, Pass RF, et al. Hearing loss in children with congenital cytomegalovirus infection born to mothers with preexisting immunity. J Pediatr. 2006;148(3):332-6.

12. Kenneson A, Cannon MJ. Review and meta-analysis of the epidemiology of congenital cytomegalovirus (CMV) infection. Rev Med Virol. 2007;17(4):253-76.

13. Lazzarotto T, Guerra B, Lanari M, Gabrielli L, Landini MP. New advances in the diagnosis of congenital cytomegalovirus infection. J Clin Virol. 2008;41(3):192-7.

14. Rutter D, Griffiths P, Trompeter RS. Cytomegalic inclusion disease after recurrent maternal infection. Lancet. 1985;2:1182-6.

15. Ahlfors K, Harris S, Ivarsson S, Svanberg L. Secondary maternal cytomegalovirus infection causing symptomatic congenital infection. N Engl J Med. 1981;305:284

16. Wang G, Zhang X, Bialek S, Cannon MJ. Attribution of congenital cytomegalovirus infection to primary versus non-primary maternal infection. Clin Infect Dis. 2011;52:e11-3.

17. de Vries JJ, Zwet EW, Dekker FW, Kroes AC, Verkerk PH, Vossen AC. The apparent paradox of maternal seropositivity as a risk factor for congenital cytomegalovirus infection: a population-based prediction model. Rev Med Virol. 2013;23:241-9.
18. Stagno S, Reynolds DW, Huang ES, Thames SD, Smith RJ, Alford CA. Congenital cytomegalovirus infection. N Engl J Med. 1977;296(22):1254-8.

19. Schopfer K, Lauber E, Krech U. Congenital cytomegalovirus infection in newborn infants of mothers infected before pregnancy. Arch Dis Child. 1978;53(7):536-9.

20. Fowler KB, Stagno S, Pass RF. Maternal age and congenital cytomegalovirus infection: screening of two diverse newborn populations, 1980-1990. J Infect Dis. 1993;168:552-6.

21. Hadar E, Salzer L, Dorfman E, Amir J, Pardo J. Antenatal risk factors for symptomatic congenital CMV disease following primary maternal CMV infection. J Perinat Med. 2016:44(3):339-44.

22. Kyriazopoulou V, Bondis J, Frantzidou F, Athanasiadis A, Diza E, Simitsopoulou $M$, et al. Prenatal diagnosis of fetal cytomegalovirus infection in seropositive pregnant women. Eur J Obstet Gynecol Reprod Biol. 1996:69(2):91-5.

23. Zalel Y, Gilboa Y, Berkenshtat M, Yoeli R, Auslander R, Achiron R, et al. Secondary cytomegalovirus infection can cause severe fetal sequelae despite maternal preconceptional immunity. Ultrasound Obstet Gynecol. 2008;31(4):417-20

24. Huang ES, Alford CA, Reynolds DW, Stagno S, Pass RF. Molecular epidemiology of cytomegalovirus infections in women and their infants. N Engl J Med. 1980;303(17):958-62.

\section{Submit your next manuscript to BioMed Central and we will help you at every step:}

- We accept pre-submission inquiries

- Our selector tool helps you to find the most relevant journal

- We provide round the clock customer support

- Convenient online submission

- Thorough peer review

- Inclusion in PubMed and all major indexing services

- Maximum visibility for your research

Submit your manuscript at www.biomedcentral.com/submit
Biomed Central 